\title{
Introduction to Category Theory
}

\section{Olivier Bousquet}

Department of Empirical Inference

Max Planck Institute of Biological Cybernetics

olivier. bousquet@tuebingen.mpg. de

8th of January 2004 


\section{Informal Description}

- "Generalized mathematical theory of structures"

- Goal: "reveal the universal properties of structures via their relationships"

- Emphasis on relationships rather than on objects

- Uniform treatment of the notion of structure

- Provides new foundations to mathematics (debated)

- Many applications in mathematics, mathematical physics, computer science...

O. Bousquet - Introduction to Category Theory 


\section{Definition}

A category is given by

- Collection of objects

- For each pair of objects $a, b$, collection of morphisms (or arrows) $\operatorname{Mor}(a, b)$

- Composition: $\operatorname{Mor}(a, b) \times \operatorname{Mor}(b, c) \rightarrow \operatorname{Mor}(a, c)$

- Identity: $i d_{a} \in \operatorname{Mor}(a, a)$

Axioms

- Associativity of composition: $f \circ(g \circ h)=(f \circ g) \circ h$

- Identity: $\left(i d_{a} \circ f\right)=\left(f \circ i d_{b}\right)=f$ 


\section{Examples (I)}

- Sets with functions

- Groups with group homomorphisms

- Topological spaces with continuous maps

- Vector spaces with linear maps

- Differentiable manifolds with smooth maps

$\rightarrow$ Typical: objects are structured sets and morphisms are structure preserving maps

O. Bousquet - Introduction to Category Theory 


\section{Examples (II)}

But categories are more general than this:

- Preordered set: elements and comparisons

- Group: 1 object and morphisms are group elements

- Directed graph: objects are vertices and morphisms are paths 


\section{Universality (I)}

Unification of mathematical structures: factorization of recurring constructions.

Product

- Given two objects $a, b$ in $C$

- The product is the triple $(c, p, q)$

- $c$ object of $C$ (representing the "cartesian product $a \times b^{\prime \prime}$ )

- $p: c \rightarrow a$ and $q: c \rightarrow b$ morphisms (representing "projections")

- Universality property: for all $d \in C$ and $f: d \rightarrow a, g: d \rightarrow b, \exists$ ! morphism $h: d \rightarrow c$ s.t. $p \circ h=f$ and $q \circ h=g$ 


\section{Examples}

- Sets: cartesian product

- Groups: product of groups (with appropriate group structure)

- Preordered set: least upper bound

- Exercise: product in a graph ?

$\rightarrow$ Because emphasis on morphisms, structures are automatically transported to products

$\rightarrow$ Notion of smallest product included in the universality property 


\section{Diagrams}

- Convenient way of representing statements

- Commutation means equality of paths

- Example: axioms of categories (associativity, unity), product

$\rightarrow$ See whiteboard!

O. Bousquet - Introduction to Category Theory 


\section{Relationships: Functors}

Unification of mathematical structures: study of relationships between structures

- A functor is a morphism of categories (preserves structure)

- $F: C \rightarrow D$ maps objects of $C$ to objects of $D$ and morphisms to morphisms

- $f: c \rightarrow c^{\prime}$ is mapped to $F(f): F(c) \rightarrow F\left(c^{\prime}\right)$

- $F\left(i d_{c}\right)=i d_{F(c)}$

- $F(f \circ g)=F(f) \circ F(g)$

O. Bousquet - Introduction to Category Theory 


\section{Examples}

- Power set functor $P:$ Set $\rightarrow$ Set

$\star$ Maps $X$ to $P(X)$ set of subsets of $X$

$\star$ Maps $f: X \rightarrow Y$ to $P(f): S \subset X \mapsto f(S) \subset Y$

- Linear group of invertible matrices $G L_{n}: C R i n g \rightarrow G r p$

- Homotopy group Top $\rightarrow$ Grp

- Forgetful Grp $\rightarrow$ Set 


\section{Relationships: Natural transformations}

Relationships between functors $F, G: C \rightarrow D$ (morphism of functors)

- For each $c \in C$, consider a morphism $h_{c}: F(c) \rightarrow G(c)$

- Commutativity condition: $f: c \rightarrow d, G(f) \circ h_{c}=h_{d} \circ F(f)$

$\rightarrow$ Diagram on whiteboard 


\section{Examples}

- Determinant (morphism from complex to real rings, $F$ is $G L_{n}$ and $G$ is group of invertible elements)

- Identity to power set 


\section{Limits}

- More general than products

- Given categories $C, J$ ( $J$ is the index set) and functor $F: J \rightarrow C$ (defines which objects are used in the "product")

- Define limit object $r \in C$

- For each $c \in C, j \in J$, a morphism $h_{c j}: c \rightarrow F(j)$

- Universality property

$\rightarrow$ Product are a special case with $J=\{1,2\}$ 


\section{Adjoint Functors}

- More fundamental than limits, cornerstone of the theory

- Categories $C, D$ and functors $F: C \rightarrow D, G: D \rightarrow C$

- In addition, a map $\phi: O b(C) \times O b(D) \rightarrow$ bijections of morphisms $M \operatorname{or}_{D}(F(c), d) \equiv \operatorname{Mor}_{C}(c, G(d))$

- Commutativity property 


\section{Examples}

- Set, $V c t, F$ free vector space, $G$ forgetful functor, $\phi(S, V)$ bijection between linear maps $F(S) \rightarrow V$ and maps $S \rightarrow G(V)$

- $C \times C, C, F$ product functor, $G$ diagonal functor $(c \mapsto(c, c))$

- $C^{J}, C, F$ limit functor, $G$ diagonal functor

- $\{1\}, C, F$ terminal object, $G$ diagonal functor 


\section{Relationship with Set Theory}

- In set theory, identify equal elements

- In CT, identify isomorphic elements

- In CT, classes of objects need not be sets

- Category of all sets is defined even if there is no set of all sets 


\section{Higher Dimensional}

Climb the category theoretic ladder to investigate more relationships Start with notion of cells (diagram)

- 0-cells: points

- 1-cells: arrows

- 2-cells: arrows between arrows

- 3-cells: ... 


\section{n-categories}

- 0-categories: sets

- 1-categories: standard categories

- 2-categories: e.g. Cat with categories, functors and natural transformations

- More general example: topological spaces and paths, maps between paths... 


\section{Axioms}

- Appropriate properties for cell relationships (iteratively using definition of categories)

- In 2-categories: e.g. horizontal and vertical composition with $\left(\alpha \alpha^{\prime}\right) \otimes\left(\beta \beta^{\prime}\right)=(\alpha \otimes \beta)\left(\alpha^{\prime} \otimes \beta^{\prime}\right)$

- Replace equality by isomorphism: weak $n$-categories (more interesting) but need coherence relationships

- Unfortunately, combinatorial effects (which equalities to weaken ?) prevents from easily finding general definition

$\rightarrow$ Major topic of research in foundations of mathematics, mathematical physics (topological quantum field theory) and philosophy 